\title{
Caracterización Y perfil lipídico de aceites de microalgas
}

\author{
Characterization and lipid profile of oil from microalgae \\ Caracterização e perfil lipídico de azeites de microalgas
}

Fecha de Recepción: 05 de Septiembre de 2014

Fecha de Aceptación: 12 de Enero de 2015

\author{
Lesly Tejeda-Benítez* \\ Daniela Henao-Argumedo** \\ Mario Alvear-Alayón*** \\ Carlos Rafael Castillo-Saldarriaga****
}

\section{Resumen}

El uso creciente del biodiésel ha impulsado la búsqueda de nuevas materias primas, dentro de las que se destacan las microalgas. En esta investigación se estudió el cultivo de las microalgas Chlorella sp. y Dunaliella salina bajo diferentes condiciones de $\mathrm{pH}$ y concentración de nitrógeno $\left(\mathrm{mgL}^{-1}\right)$, y la caracterización de los aceites obtenidos, con el fin de evaluar su potencial uso como materia prima en la producción de biodiésel. La $D$. salina alcanzó una máxima concentración celular de $1.15 \times 10^{6}$ células $\mathrm{mL}^{-1}$ en 6 días de cultivo en unas condiciones de $8.5 \mathrm{pH}$ y $0.1 \mathrm{mg} \mathrm{L}^{-1}$ concentración de nitrógeno, mientras que Chlorella $s p$. presentó una máxima concentración celular de $2.6 \times 10^{7}$ células $\mathrm{mL}^{-1}$ en 14 días de cultivo en unas condiciones de $7.5 \mathrm{pH}$ y $0.1 \mathrm{mg} \mathrm{L}^{-1}$ concentración de nitrógeno. La extracción de aceite intracelular de las microalgas cultivadas bajo las mejores condiciones de crecimiento celular se realizó empleando el método modificado de Bligh \& Dyer. Al determinar el perfil de ácidos grasos de los aceites extraídos de ambas microalgas, se encontraron en mayor proporción dos ácidos grasos insaturados: ácido linolénico y ácido oleico. En el aceite de D. salina se encontró una concentración de $51 \%$ p/p de ácido linolénico, mientras que para el aceite de Chlorella $s p$. fue de 39\% $\mathrm{p} / \mathrm{p}$. Con relación al ácido oleico, el aceite de Chrorella sp. presentó una concentración de $35 \% \mathrm{p} / \mathrm{p}$, superior al aceite de D. salina, con $25 \% \mathrm{p} / \mathrm{p}$.

Palabras clave: Ácidos grasos, Biodiésel, Lípidos, Microalgas.

\footnotetext{
* Ph.D. (c). Universidad de Cartagena (Cartagena - Distrito Turístico, Colombia). 1tejedab@unicartagena.edu.co.

** M.Sc. (c). Universidad de Cartagena (Cartagena - Distrito Turístico, Colombia). danielahenaoa@hotmail.com.

*** M.Sc. (c). Universidad de Cartagena (Cartagena - Distrito Turístico, Colombia).malvearaa@gmail.com.

**** M.Sc. (c). Universidad Nacional de Colombia (Bogotá - Distrito Capital, Colombia). crcastillos@unal.edu.co.
} 


\section{Abstract}

The increasing use of biodiesel has prompted the search for new raw materials, among them are highlighted the microalgae. In this research the cultivation of microalgae Chlorella sp. and Dunaliella salina at different conditions of $\mathrm{pH}$ and nitrogen is reported; and the oils obtained from them characterization, in order to assess the biodiesel potential production. D. salina reached the maximun cells concentration, during a 6 days cultivation, at $\mathrm{pH} 8.5$ and $0.1 \mathrm{mg} \mathrm{L}^{-1}$ of nitrogen, obtaining values of $1.15 \times 10^{6}$ cells $\mathrm{mL}^{-1}$, while the Chlorella sp. under a $\mathrm{pH}$ value of 7.5 and $0.1 \mathrm{mg} \mathrm{L}^{-1}$ of nitrogen conditions, achieved the maximun concentrations of $2.6 \times 10^{7} \mathrm{cells}^{-1}$. Analysis of the fatty acid profiles revealed that the most abundant were linolenic, and then, oleic; this implies a large number of unsaturations that would have negative implications on the physicochemical properties and performance of biodiesel combustion.

Keywords: Fatty Acids, Biodiésel, Lipids, Microalgae.

\section{Resumo}

O uso crescente do biodiesel tem impulsado a busca de novas matérias primas, dentro das quais se destacam as microalgas. Nesta pesquisa se estudou o cultivo das microalgas Chlorella sp. e Dunaliella salina sob diferentes condições de $\mathrm{pH}$ e concentração de nitrogênio $\left(\mathrm{mgL}^{-1}\right)$, e a caracterização dos azeites obtidos, com o fim de avaliar seu potencial uso como matéria prima na produção de biodiesel. A D. salina alcançou uma máxima concentração celular de $1.15 \times 10^{6}$ células $\mathrm{mL}^{-1} \mathrm{em} 6$ dias de cultivo em umas condições de $8.5 \mathrm{pH} \mathrm{e} 0.1 \mathrm{mg} \mathrm{L}^{-1}$ concentração de nitrogênio, enquanto que Chlorella sp. apresentou uma máxima concentração celular de $2.6 \times 10^{7}$ células mL-1 em 14 dias de cultivo em umas condições de 7.5 pH e $0.1 \mathrm{mg} \mathrm{L}^{-1}$ concentração de nitrogênio. A extração de azeite intracelular das microalgas cultivadas sob as melhores condições de crescimento celular se realizou empregando o método modificado de Bligh \& Dyer. Ao determinar o perfil de ácidos graxos dos azeites extraídos de ambas as microalgas, encontraram-se em maior proporção dois ácidos graxos insaturados: ácido linolênico e ácido oleico. No azeite de D. salina se encontrou uma concentração de $51 \%$ p/p de ácido linolênico, enquanto que para o azeite de Chlorella sp. foi de $39 \%$ p/p. Com relação ao ácido oleico, o azeite de Chrorella sp. apresentou uma concentração de $35 \%$ p/p, superior ao azeite de D. salina, com $25 \%$ p/p.

Palavras chave: Ácidos graxos, Biodiesel, Lípidos, Microalgas.

Cómo citar este artículo:

[1] L. Tejeda-Benítez, D. Henao-Argumedo, M. Alvear-Alayón \& C.R. Castillo-Saldarriaga, "Caracterización y perfil lipídico de aceites de microalgas", Fac. Ing., vol. 24 (39), pp. 43-54, Mayo-Ago. 2015. 


\section{INTRODUCCIÓN}

La búsqueda de combustibles sostenibles y renovables cobra cada vez más importancia como resultado directo del cambio climático y del aumento de los precios del petróleo. Uno de los combustibles de mayor interés es el biodiésel o éster metílico de ácidos grasos, cuya producción implica la transesterificación de ácidos grasos por catálisis alcalina en presencia de un alcohol [1]; en la actualidad, estos ácidos grasos se extraen de plantas oleoginosas, como la palma de aceite, desviando la cadena productiva de aceites hacia la producción de biocombustibles, con lo cual se crea un riesgo sobre la seguridad alimentaria de los países en vía de desarrollo. Adicionalmente, cultivos energéticos, como la palma de aceite, requieren grandes áreas de tierra cultivable, y son demandantes de una alta cantidad de agua dulce empleada en sistemas de riego [1].

Debido a lo anterior, ha surgido la necesidad de buscar nuevas fuentes de aceites que no requieran grandes cantidades de tierra y hagan un mejor uso del agua; una de las opciones ha sido el aceite de microalgas, microorganismos que son capaces de convertir de manera eficiente el $\mathrm{CO}_{2}$ y la luz solar en biomasa y aceites intracelulares, y que al no ser usados como fuente primaria de alimentos para humanos evitan las preocupaciones por la seguridad alimentaria. Incluso en un escenario conservador, se estima que el uso de las microalgas producirá cerca de 10 veces más biodiésel por unidad de superficie de tierra que un típico oleaginosa terrestre [1-6].

Las microalgas están compuestas, básicamente, por proteínas, carbohidratos, ácidos nucleicos y ácidos grasos. Los ácidos grasos se encuentran en las membranas, en los productos de almacenamiento, metabolitos, etc. En algunas especies, los ácidos grasos representan el $40 \%$ de su peso seco. Para la producción de biodiésel se requieren microalgas que contengan un alto contenido en lípidos y que sean fácilmente cultivables. Las mejores algas para producir biodiésel son las microalgas con un diámetro menor a $2 \mathrm{~mm}$, ya que producen más aceite y crecen de forma más rápida y fácil que las macroalgas $[3,6]$.

El contenido de lípidos de las microalgas varía considerablemente de una especie a otra, y puede variar, en términos de biomasa seca, entre el 5 y el $77 \%$ en peso. El perfil de ácidos grasos y la composición de los lípidos extraídos de una especie en particular se ve afectada por el ciclo de vida y las condiciones de cultivo, tales como la composición del medio, la temperatura, la intensidad de iluminación, la relación del ciclo de luz/oscuridad y la tasa de aireación [1, 4]. Algunas especies de microalgas pueden aumentar su contenido de lípidos entre un 10 al $20 \%$ en peso durante períodos de escasez de oxígeno. Las microalgas, generalmente, responden a la ausencia de nutrientes mediante la intensificación de la vía metabólica, que sintetiza lípidos neutros; sin embargo, este aumento de la producción de lípidos no ocasiona, por lo general, un aumento de la productividad de aceite por unidad de masa, ya que se realiza a menudo a costa de sacrificar la tasa de crecimiento y, por ende, la división celular. No obstante, el contenido de aceite por sí solo no determina la productividad; la Botryococcus braunii puede alcanzar niveles de aceite del 75\%, pero tiene una baja productividad de biomasa [5], y las microalgas más comúnmente usadas, como la Chlorella vulgaris, tienen contenido de aceite más bajos, pero alcanza productividades más altas $[7,8]$. Debido a lo anterior, para la producción de biodiésel se requiere optimizar la producción de lípidos, examinando cada caso por separado, para establecer así las mejores condiciones de cultivo $[3,6]$.

Una diferencia importante entre los ésteres metílicos derivados de microalgas y los de aceites vegetales es que algunos lípidos de algas contienen una cantidad sustancial de ácidos grasos poliinsaturados de cadena larga, los cuales son mejores que los ésteres metílicos de ácidos grasos en términos de las propiedades del combustible, incluyendo el número de cetano, estabilidad a la oxidación y propiedades de flujo en frío [9].

La composición de ácidos grasos de las diferentes especies de microalgas también es importante, porque puede tener un efecto significativo sobre la calidad del biodiésel producido; estos se componen de saturados e insaturados, con 12-22 átomos de carbono. Estudios sobre diferentes especies de microalgas de agua dulce han encontrado que en la mayoría de ellas se encuentran ácidos grasos C14:0, C16:0, C18:1, C18:2 y C18:3. Los factores 
nutricionales y ambientales y las condiciones del cultivo pueden afectar la tasa de crecimiento de biomasa y la composición de ácidos grasos; por ejemplo, la deficiencia de nitrógeno y el estrés salino inducen la acumulación de C18:1 [10].

Existen varios obstáculos tecnológicos y económicos para la producción a escala industrial de biodiésel a partir de aceite de microalgas; algunas de estas están relacionados con la velocidad de crecimiento, el rendimiento y la productividad del cultivo de microalgas; además, se requiere identificar las condiciones de cultivo que incentiven la producción y acumulación intracelular de ácidos grasos, y superar las limitaciones tecnológicas relacionadas con las operaciones de cosecha y extracción [1-6].

Para la selección de cepas de microalgas con el fin de obtener ácidos grasos de calidad para la producción de biodiésel se debe tener en cuenta la influencia de condiciones de crecimiento sobre parámetros cinéticos como: capacidad de acumular ácidos grasos saturados, concentración celular y productividad. Por lo anterior, en este trabajo se realizó la evaluación de dos factores: $\mathrm{pH}$ y concentración de nitrógeno, para determinar su influencia sobre el crecimiento de dos cepas de microalga: Chlorella sp., y Dunaliella salina, con un posterior análisis de las propiedades fisicoquímicas y perfil de ácidos grasos de los aceites extraídos para evaluar su calidad como materia prima para la producción de biodiésel.

\section{Materiales y MÉTOdos}

\section{A. Cepas de microalgas}

Para el estudio se emplearon dos cepas de microalgas: Chlorella sp., aislada de la Bahía de Cartagena en los laboratorios del Centro Internacional Náutico Fluvial y Portuario SENA, y D. salina, adquirida del Laboratorio de Biotecnología de la Universidad de Antioquia.

\section{B. Mantenimiento de microalgas}

Las microalgas se conservaron en agar Walne [13], a una temperatura de $26 \pm 2{ }^{\circ} \mathrm{C}$, una intensidad lumínica de 300 lux y un fotoperíodo de 24 horas.

\section{Cultivo de microalgas}

Cada 15 días se realizó un recambio del medio para conservar las cepas en fase de crecimiento, y se pasó una muestra renovada a un volumen efectivo de trabajo de $300 \mathrm{ml}$ agitados por aire procedente de un motor de aireación; para luego, en un par de días, iniciar montaje de los ensayos con un $25 \%(\mathrm{v} / \mathrm{v})$ de estos inóculos. Para los ensayos experimentales se realizó un diseño experimental factorial completo $2^{2}$, en el cual los factores de interés estudiados fueron la concentración inicial de nitrógeno y $\mathrm{pH}$. El medio de cultivo empleado para el crecimiento de las microalgas fue Conwy modificado. El cultivo se realizó en recipientes con un volumen efectivo de $4 \mathrm{~L}$ y un volumen de trabajo de $3 \mathrm{~L}$ (ver Figura 1), acoplados a un sistema de aireación. Para el crecimiento de la cepa Chlorella sp. se empleó agua desionizada estéril, mientras que para la cepa $D$. salina se utilizó agua de mar filtrada estéril.

Para ajustar la concentración de nitrógeno en los ensayos se empleó nitrato de sodio $\left(\mathrm{NaNO}_{3}\right)$. $\mathrm{El} \mathrm{pH}$ se estableció con soluciones de $0.1 \mathrm{M}$ de hidróxido de sodio $(\mathrm{NaOH})$ o ácido clorhídrico $(\mathrm{HCl})$, según fuera el caso. Se realizaron recuentos cada 24 horas en cámara de Neubauer. El tiempo de crecimiento para la cepa de Chlorella sp. fue de 11 días, mientras que para la cepa $\mathrm{D}$. salina fue de 25 días $[11,12]$.

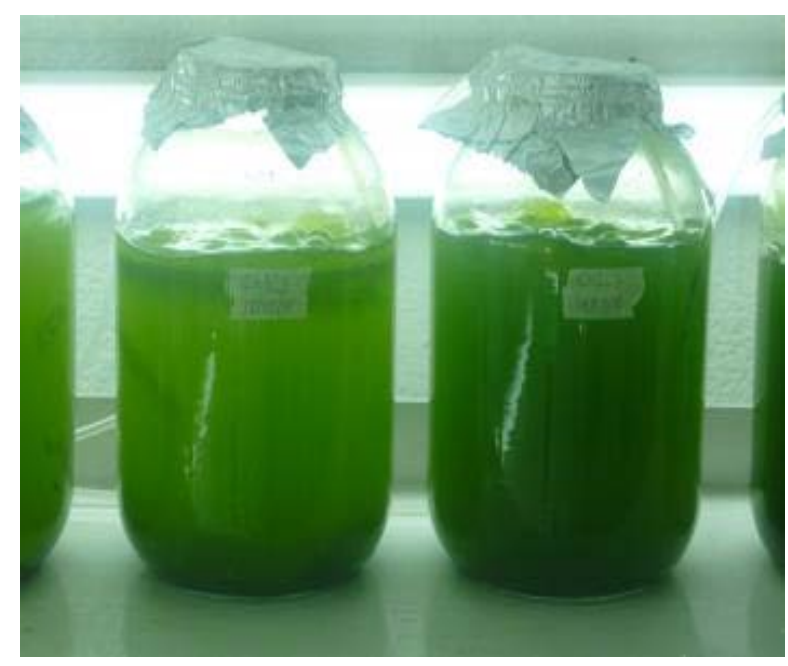

Fig. 1. Cultivos de Chlorella sp. y D. salina en frascos de $4 \mathrm{~L}$ 


\section{Recolección de biomasa}

Con las mejores condiciones de crecimiento para cada microalga, se realizaron cultivos a escala de 20 L. Con base en la cinética de crecimiento reportada de las mediciones del conteo celular, fue realizada la separación de la biomasa de las microalgas durante la fase estacionaria, mediante el filtrado de las muestras y posterior secado en un horno a $105{ }^{\circ} \mathrm{C}$ durante una hora.

\section{E. Extracción y caracterización de aceites}

Para extraer los aceites totales presentes en la biomasa microalgal se usó el método modificado de Bligh \& Dyer, que consiste en la homogeneización, a 800 rpm, de la biomasa con una mezcla de metanol cloroformo en proporción $2: 1$, seguido de la adición de cloroformo y homogeneización durante 30 s, con posterior adición de agua y homogeneización durante 30 s adicionales. Posteriormente se realiza una filtración y una centrifugación; después de la cual se separan las fases de metanol y cloroformo, y se remueve el cloroformo por evaporación [14]. La Fig. 2 muestra el proceso de extracción del aceite. Los aceites extraídos fueron caracterizados mediante las técnicas reportadas por la Norma Técnica Colombiana (NTC) para grasas y aceites vegetales y animales, y se determinó índice de acidez [15], índice de peróxido [16], humedad y materia volátil [17], índice de refracción [18], densidad [19] y punto de humo [20].

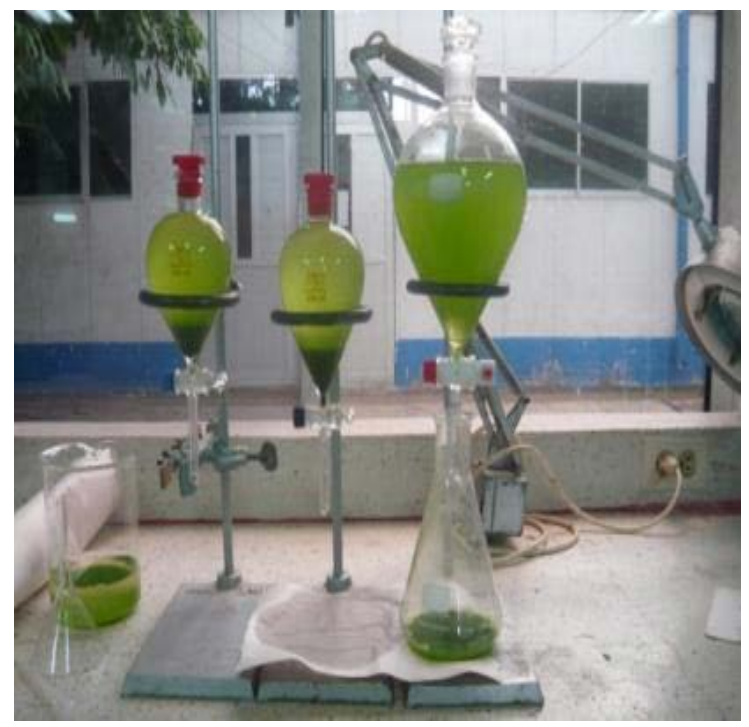

Fig. 2. Extracción de aceites de microalgas

\section{F. Perfil de ácidos grasos}

Los aceites extraídos de los cultivos de 20 L fueron evaluados para determinar el contenido de ácidos grasos mediante cromatografía gas-líquido, usando columnas capilares de fase reversa y empleando como agente esterificante hidróxido de potasio en metanol.

\section{G. Análisis estadístico}

Los datos fueron procesados mediante un análisis estadístico con la herramienta del ANOVA multifactorial, donde el número promedio de células alcanzado para cada ensayo de las microalgas Chlorella sp. y D. salina fue comparado con respecto a las variables de estudio, concentración de nitrógeno y $\mathrm{pH}$.

\section{RESULTADOS Y DISCUSIÓN}

\section{A. Curvas de crecimiento}

En la Fig. 3 se muestran las curvas de crecimiento de los cultivos de Chlorella sp. bajo las diferentes condiciones de concentración de nitrógeno y $\mathrm{pH}$. Los valores obtenidos del conteo celular para todos los ensayos presentan picos máximos de crecimiento, en el rango entre $2 \times 10^{7}$ y $2.6 \times 10^{7}$ células $\mathrm{mL}^{-1}$, y tasas de crecimiento entre 0.427 y 0.461 divisiones día ${ }^{-1}$. Los máximos valores alcanzados se obtuvieron con una concentración de nitrógeno de $0.1 \mathrm{mg} \mathrm{L}^{-1} \mathrm{y} \mathrm{pH}$ de 7.5. Este resultado es superior en dos órdenes de magnitud al obtenido por [21] con Chlorella vulgaris, cuya concentración máxima fue de $1 \times 10^{5}$ células $\mathrm{mL}^{-1}$ para cultivos con una concentración de nitrógeno de $8.4 \mathrm{mg}$ $\mathrm{L}^{-1} \mathrm{y}$ un $\mathrm{pH}$ de 6.4. De las curvas se puede observar cómo para la Chlorella sp., a mayor valor de $\mathrm{pH}$ se presenta un bajo crecimiento celular, manteniendo igual la concentración de nitrógeno. Lo anterior puede fundamentarse de acuerdo con lo reportado por [22], quienes argumentaron que para la Chlorella vulgaris un valor de $\mathrm{pH}$ elevado originará un menor consumo de oxígeno, alterando así el crecimiento celular. 


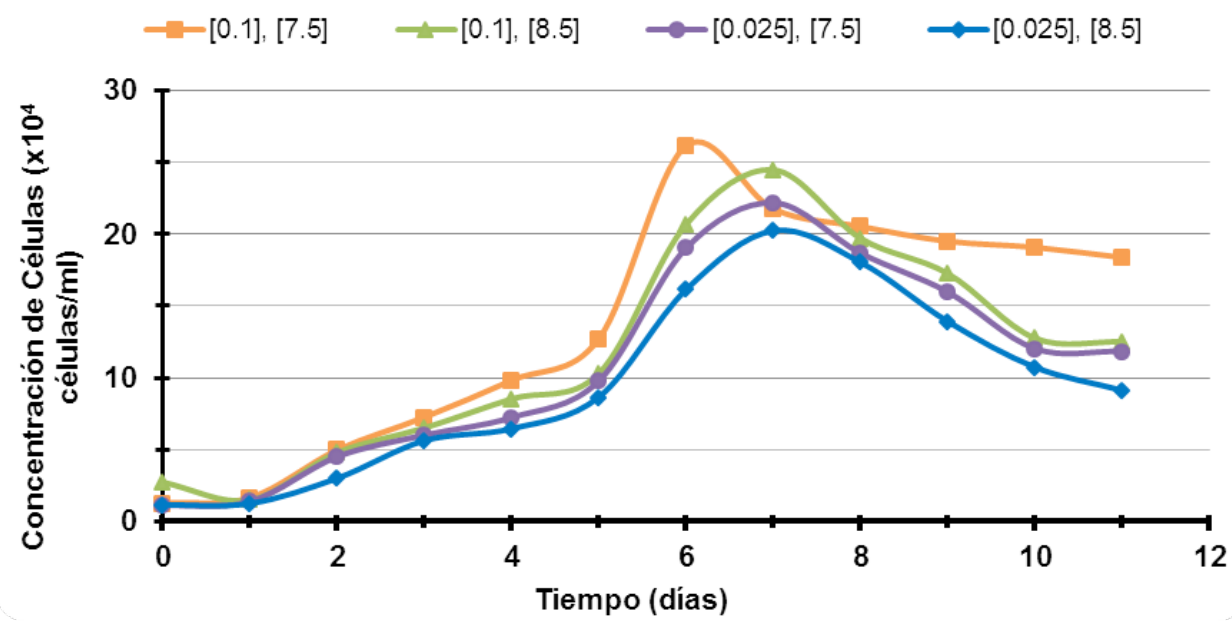

Fig. 3. Curva de crecimiento de Chlorella sp. Los números en corchetes corresponden a la concentración de nitrógeno y $\mathrm{pH}$, respectivamente. El valor reportado es el promedio de las mediciones de las dos repeticiones.

Por otra parte, en la Fig. 4 se presentan las curvas de crecimiento de los cultivos de D. salina evaluadas bajo las mismas condiciones de experimentación de los cultivos de Chlorella sp. Los valores obtenidos del conteo celular presentaron picos máximos de crecimiento, en el intervalo de $8.2 \times 10^{5}$ y $1.15 \times 10^{6}$ células $\mathrm{mL}^{-1}, \mathrm{y}$ tasas de crecimiento entre 0.075 y 0.093 divisiones día ${ }^{-1}$, respectivamente. Donde los valores máximos se alcanzaron para la mayor concentración de nitrógeno y el mayor valor de $\mathrm{pH}$ analizado (0.1 $m g \mathrm{~L}^{-1}$ y 8.5 , respectivamente). Estos resultados concuerdan con lo reportado por [23], que obtuvieron tasas de crecimiento de 0,066 y 0,082 divisiones/día a
$\mathrm{pH}$ de 7.0 y 8.0, respectivamente. La densidad celular encontrada concuerda con lo reportado por [24] y [25], quienes alcanzaron concentraciones de $1.2 \times 10^{6}$ y $1.6 \times 10^{6}$ células $\mathrm{mL}^{-1}$, respectivamente. En general, se observa cómo para esta microalga la asimilación de altas concentraciones de nitratos se encuentra estrechamente relacionada con el $\mathrm{pH}$, puesto que entre menor sea la concentración de nitrógeno presente, mucho más fácil será su asimilación; mientras que a mayores concentraciones son necesarios valores suficientemente básicos ( $\mathrm{pH}>8.0)$. Estos valores de $\mathrm{pH}$ fueron reportados por [24], quienes encontraron pH óptimos entre 8.7 y 9.

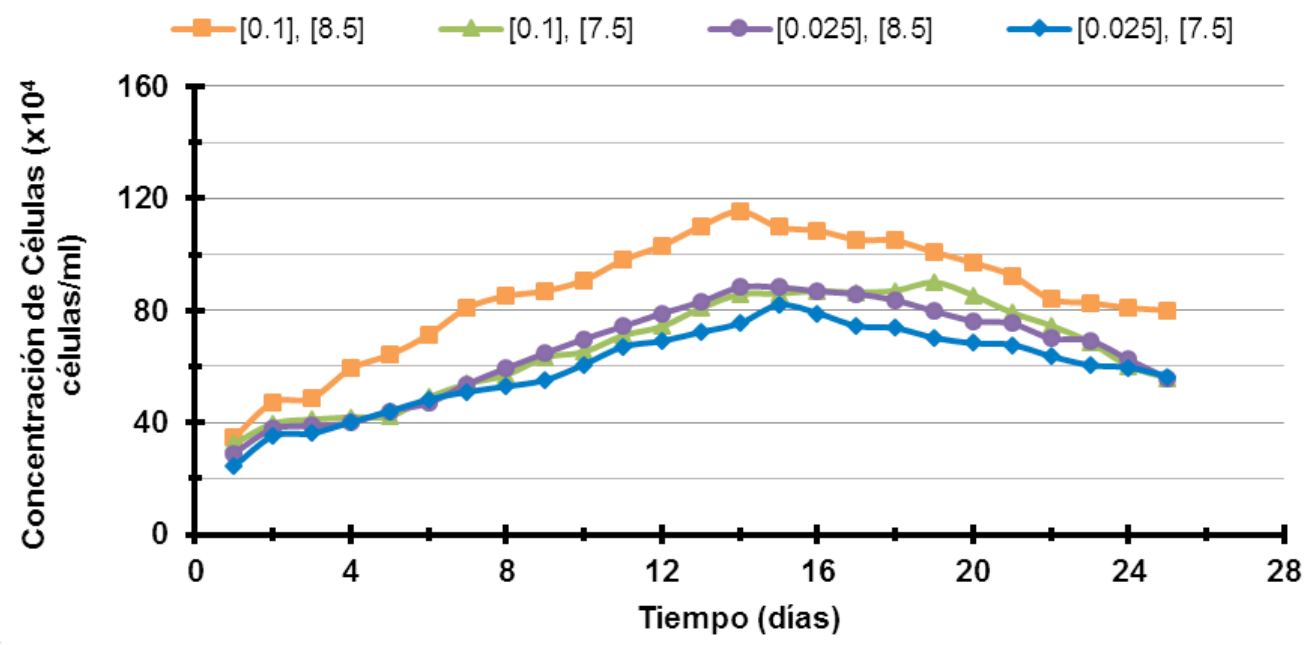

Fig. 4. Curva de crecimiento de D. salina. Los números en corchetes corresponden a la concentración de nitrógeno y $\mathrm{pH}$, respectivamente. El valor reportado es el promedio de las mediciones de las dos repeticiones. 
Del análisis estadístico se concluye que para las dos microalgas, la concentración de nitrógeno, el valor de $\mathrm{pH}$ y la interacción entre estos tienen incidencia en la densidad celular $(\mathrm{p}<0.05)$.

\section{B. Productividad de lípidos}

La viabilidad para obtener biodiésel a partir de aceite de microalga depende de la productividad para producir biomasa y de cuánta de esta biomasa corresponde a lípidos. En este trabajo se encontró producción de biomasa de $98.61 \mathrm{mg} \mathrm{L}^{-1}$ día $^{-1}$ para la Chlorella sp., y $71.89 \mathrm{mg} \mathrm{L}^{-1}$ día $^{-1}$ para la $D$. salina; sin embargo, el contenido de lípidos se ve favorecido ampliamente en el cultivo de Chlorella sp., con el $40.23 \%$, en comparación con el $23.48 \%$ alcanzado con la biomasa de D. salina .
Los resultados del presente trabajo son comparables con los obtenidos en otras investigaciones [26-29]; sin embargo, vale la pena anotar que el contenido de lípidos en la biomasa depende de las condiciones del cultivo, que no son las mismas que las reportadas, y de la fase de crecimiento en la cual fueron cosechadas las microalgas, puesto que, por lo general, el crecimiento de microalgas y la acumulación de lípidos no ocurren en forma paralela en el cultivo. La Tabla 1 muestra la productividad de lípidos para otros tipos de Chlorella. Se puede observar que la $C$. vulgaris aparece en varios trabajos reportados con una alta productividad de biomasa, pero al incluir el contenido de lípidos, la Chlorella sp. de este estudio alcanza una productividad de lípidos ligeramente mayor. Por otro lado, estudios con Chlorella de cultivos mixotróficos han mostrado altas tasas de crecimiento, en comparación con los autótrofos o heterótrofos [28].

TABLA 1

Productividad de BIOMASA Y LíPIDOS DE DIFERENTES TIPos DE ChLORELla

\begin{tabular}{|c|c|c|c|c|}
\hline Microalga & $\begin{array}{l}\text { Productividad de biomasa } \\
\qquad\left(\mathrm{mg} \mathrm{L}^{-1} \text { día }^{-1}\right)\end{array}$ & $\begin{array}{l}\text { Contenido de } \\
\text { lípidos (\%) }\end{array}$ & $\begin{array}{l}\text { Productividad de lípidos } \\
\qquad\left(\mathrm{mg} \mathrm{L}^{-1} \text { día }^{-1}\right)\end{array}$ & Ref. \\
\hline Chlorella $\mathrm{sp}$. & 98.61 & 40.23 & 39.67 & $*$ \\
\hline Chlorella vulgaris & 170 & 19.2 & 32.6 & {$[26]$} \\
\hline Chlorella vulgaris & 200 & 18.4 & 36.9 & {$[26]$} \\
\hline $\begin{array}{l}\text { Chlorella vulgaris } \\
\text { fototrófica }\end{array}$ & 10 & $33-38$ & 4 & {$[27]$} \\
\hline $\begin{array}{l}\text { Chlorella vulgaris } \\
\text { heterotrófica }\end{array}$ & 80 & $23-36$ & $27-35$ & {$[27]$} \\
\hline $\begin{array}{l}\text { Chlorella vulgaris } \\
\text { mixotrófica }\end{array}$ & 90 & $21-34$ & $22-54$ & {$[27]$} \\
\hline $\begin{array}{l}\text { Chlorella } \\
\text { sp.mixotrópica }\end{array}$ & 363 & N. D. & N. D. & {$[28]$} \\
\hline Chlorella sp. & 129 & 31.12 & 53.96 & [29] \\
\hline Chlorella sp. & 58.4 & 32 & 17.99 & {$[30]$} \\
\hline
\end{tabular}

* Este trabajo

Del mismo modo, la Tabla 2 presenta la productividad de lípidos para la $D$. salina de este trabajo, y la $D$. tertiolecta de otros reportes, dado que no hay muchos estudios sobre D. salina. Entre las especies de Dunaliella mostradas, la D. Salina aparece con la menor productividad de biomasa; sin embargo, su productividad de lípidos es igual a la de la $D$. tertiolecta reportada por [31]. 


\section{TABLA 2}

Productividad De BIOMASA Y LíPIDOS DE DIFERENTES TIPOS DE DunAliella

\begin{tabular}{|l|c|c|c|c|}
\hline \multicolumn{1}{|c|}{ Microalga } & $\begin{array}{c}\text { Productividad de biomasa } \\
\text { (mgL }^{-1} \mathbf{d i ́}^{-1} \text { ) }\end{array}$ & $\begin{array}{c}\text { Contenido de } \\
\text { Lípidos (\%) }\end{array}$ & $\begin{array}{c}\text { Productividad de Lípidos } \\
\text { (mgL L }^{-1} \mathbf{d i ́}^{-1} \text { ) }\end{array}$ & Ref. \\
\hline Dunaliella salina & 71.89 & 23.48 & 16.88 & $*$ \\
\hline $\begin{array}{l}\text { Dunaliella } \\
\text { tertiolecta }\end{array}$ & 120 & 16.7 & 20 & {$[31]$} \\
\hline $\begin{array}{l}\text { Dunaliella } \\
\text { tertiolecta }\end{array}$ & 100 & $60.6-67.8$ & $60.6-69.8$ & {$[32]$} \\
\hline
\end{tabular}

* Este trabajo

\section{Caracterización de los aceites de microalgas}

Las caracterizaciones de los aceites de microalgas se presentan en la Tabla 3. Se reportan factores claves para la producción de biodiésel, donde para mejores rendimientos de la reacción de transesterificación se esperan valores bajos para cada uno de estos parámetros. De acuerdo con lo anterior, se observó, para los dos lípidos estudiados, bajos índices de acidez y humedad, lo que muestra una menor presencia de ácidos grasos libres, la cual será favorable para la reacción de transesterificación con $\mathrm{NaOH}$ o KOH como catalizador, produciendo menor cantidad de jabón en comparación con aceites con elevado contenido de ácidos grasos libres y agua [33].

Otras propiedades, como densidad, punto de humo e índice de refracción, resultaron muy similares entre las dos microalgas, y se encuentran dentro de los parámetros normales de cualquier aceite vegetal puro, ideal para la producción de biodiésel [34]. Por último, los aceites presentaron bajo índice de peróxido, y alto índice de yodo, lo cual favorece la oxidación; por lo tanto, se requeriría un correcto almacenamiento de los aceites sin espacios grandes de aire, poca exposición a la luz y temperaturas bajas, para evitar la degradación por oxidación.

\section{TABLA 3}

CARACTERIZACIÓN DE LOS ACEITES DE MICROALGAS

\begin{tabular}{|c|c|c|}
\hline \multirow[t]{2}{*}{ Parámetro } & \multicolumn{2}{|c|}{ Aceite de microalga } \\
\hline & D. salina & Chlorella sp. \\
\hline $\begin{array}{c}\text { Densidad } \\
\left(\mathrm{g} \mathrm{cm}^{-3}\right)\end{array}$ & 0.898 & 0.893 \\
\hline Índice de acidez & 0.513 & 0.4654 \\
\hline Humedad & 0.043 & 0.055 \\
\hline Punto de Humo & $\begin{array}{c}50^{\circ} \mathrm{C} \text { : Olor mantecoso, sin humo. } \\
100^{\circ} \mathrm{C} \text { : Olor mantecoso (menor), sin humo } \\
150^{\circ} \mathrm{C} \text { : Leve presencia de humo (color claro) } \\
200^{\circ} \mathrm{C} \text { : Leve presencia de humo color } \\
\text { amarillento. }\end{array}$ & $\begin{array}{c}50^{\circ} \mathrm{C} \text { : Olor mantecoso, sin humo. } \\
100^{\circ} \mathrm{C} \text { : Olor mantecoso (menor), sin humo } \\
150^{\circ} \mathrm{C} \text { : Leve presencia de humo (color claro) } \\
200^{\circ} \mathrm{C} \text { : Leve presencia de humo color } \\
\text { amarillento. }\end{array}$ \\
\hline Índice de refracción & 1.492 & 1.475 \\
\hline Índice de peróxido & 3.963 & 3.744 \\
\hline Índice de yodo & 164.35 & 126.79 \\
\hline
\end{tabular}




\section{Perfil de ácidos grasos}

En la Fig. 5 se muestran los resultados que arrojó el análisis de perfil de ácidos grasos de los aceites extraídos de la biomasa de Chlorella sp. y D. salina.

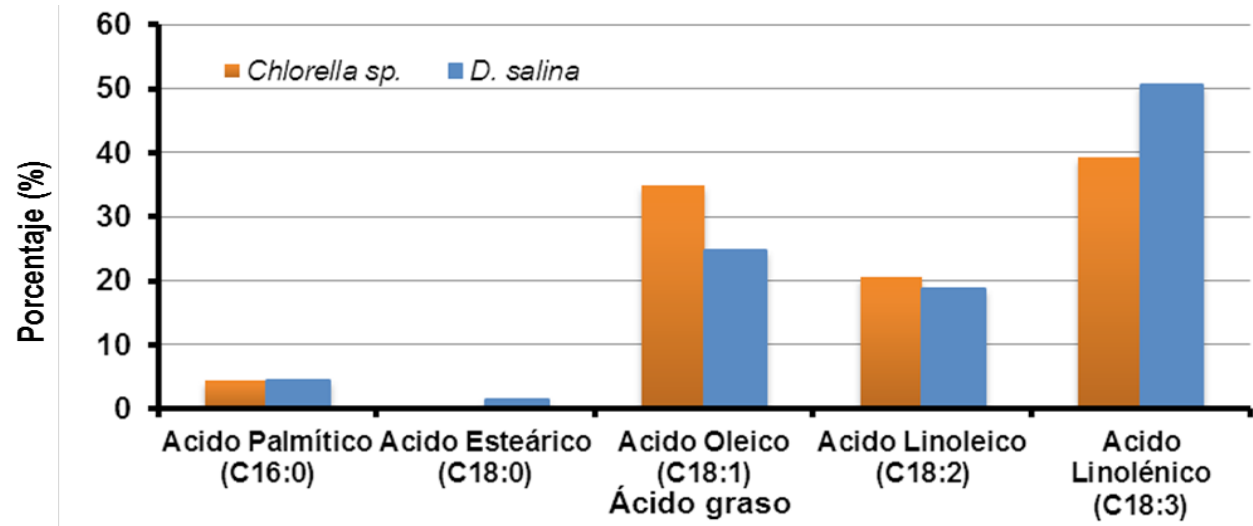

Fig. 5. Perfil lipídico de los aceites de microalgas

Como se puede observar en la Fig. 5, los aceites obtenidos a partir de las microalgas estudiadas, $\mathrm{y}$ bajo las condiciones de cultivo antes mencionadas, presenta un gran contenido de ácido oleico, ácido linoleico y ácido linolénico, que tienen en su estructura una, dos y tres insaturaciones, respectivamente. Esta característica es importante porque afecta directamente el aceite y, por lo tanto, el biodiésel producido a partir de su transesterificación. Las insaturaciones mejoran la operatividad del biodiésel a bajas temperaturas, pero disminuyen su estabilidad a la oxidación, lo cual está relacionado con el índice de yodo elevado encontrado [6]. Entre las dos especies, el aceite de la microalga $D$. salina presentó mayor contenido de ácido linolénico, que es el que más insaturaciones contiene, en comparación con aceite de la microalga Chlorella sp. Esta diferencia, junto con la mayor productividad de lípidos obtenida por la Chlorella sp. favorece la selección de esta especie para la producción de biodiésel.

La Tabla 4 muestra una comparación entre el perfil lipídico resultado de este estudio con el de otras investigaciones realizadas con Chlorella $s p$. Se observa que el contenido de cada uno de los ácidos es diferente entre las cinco investigaciones, con excepción de [24] y [28], que muestran un perfil muy similar. Estas diferencias se deben a que las condiciones a las cuales se realizaron los cultivos fueron diferentes entre sí; por ejemplo, [24] usó condiciones limitantes de nitrógeno, [28] describe un cultivo autótrofo y [29] realizó los cultivos bajo privación de nutrientes.

\section{TABLA 4}

Perfil Lipídico De DifERentes tipos De ChloRella

\begin{tabular}{|l|c|c|c|c|c|}
\hline Ácidos grasos (\%) & *Chlorella sp. & $\begin{array}{c}\text { Chlorella sp. } \\
{[\mathbf{2 4 ]}}\end{array}$ & $\begin{array}{c}\text { Chlorella sp. } \\
{[\mathbf{2 8}]}\end{array}$ & $\begin{array}{c}\text { Chlorella } \text { sp. } \\
{[\mathbf{2 9}]}\end{array}$ & $\begin{array}{c}\text { Chlorella } \text { sp. } \\
{[\mathbf{3 0}]}\end{array}$ \\
\hline Ácido palmítico & 4.45 & $23.5-26.5$ & 20.85 & 0.41 & 23.04 \\
\hline Ácido esteárico & 0.51 & $0.7-1.1$ & 0.75 & 25.94 & - \\
\hline Ácido oleico & 34.9 & $4.3-5.7$ & 7.24 & 3.03 & 21.93 \\
\hline Ácido linoleico & 20.56 & $18.8-21.2$ & 21.92 & 15.7 & 21.9 \\
\hline Ácido linolénico & 39.33 & $18.1-19.9$ & 12.79 & 0.99 & 4.27 \\
\hline
\end{tabular}

* Este trabajo 
La comparación entre el perfil lipídico del aceite extraído de $D$. salina en este estudio y el trabajo de [25] es mostrado en la Tabla 5. Se observan menores valores para el ácido palmítico; valores similares para el esteárico y linoleico, y mayores para el oleico y linolénico, diferencias que pueden estar asociadas a las condiciones de cultivo.

\section{TABLA 5}

\section{Perfil LIPÍDICO DE DIFERENTES TIPOS DE DunALIELlA}

\begin{tabular}{|l|c|c|c|c|}
\hline \multicolumn{1}{|c|}{ Ácidos grasos (\%) } & *D. salina & D. salina [25] & D. salina [25] & D. salina [25] \\
\hline Ácido palmítico & 4.44 & 21.04 & 23.32 & 24.65 \\
\hline Ácido esteárico & 1.45 & 0.20 & 0.5 & 0.62 \\
\hline Ácido oleico & 24.71 & 3.12 & 3.83 & 3.18 \\
\hline Ácido linoleico & 18.77 & 15.45 & 13.43 & 14.24 \\
\hline Ácido linolénico & 50.65 & 25.98 & 25.45 & 25.83 \\
\hline
\end{tabular}

* Este trabajo

\section{Conclusiones}

Se puede destacar que la microalga $D$. salina crece satisfactoriamente a las condiciones de cultivo de $\mathrm{pH} 8.5$, concentración de nitrógeno $0.1 \mathrm{mg} \mathrm{L}^{-1} \mathrm{y}$ salinidad alrededor de $35 \%$, con agitación continua, logrando valores superiores a los $1.15 \times 10^{6}$ células $\mathrm{mL}^{-1}$; sin embargo, esta concentración celular no es suficientemente alta para conseguir un elevado contenido en lípidos, lo que sí se logró con la Chlorella sp., con una alta velocidad de agitación de aireación, un pH inicial de 7.5 e igual concentración de nitrógeno, alcanzando concentraciones aproximadas de $2.6 \times 10^{7}$ células $\mathrm{mL}^{-1}$. De esta manera, se obtuvo un ambiente donde se presenta un factor de estrés en su crecimiento, lo que se traduce en mayor producción de lípidos. Adicionalmente, con la Chlorella sp. se alcanzó mayor productividad de lípidos que con la $D$. salina.

Con relación a las propiedades de los aceites obtenidos, se destacan los bajos índices de acidez, humedad y densidad, lo cual permite predecir un mayor rendimiento de biodiesel, al presentarse menor producción de glicerol en el proceso de transesterificación y mejores rendimientos del biodiésel obtenido mediante una combustión más completa, reduciendo principalmente emisiones de monóxido de carbono e hidrocarburos no quemados.
Pese a lo anterior, en el análisis de los perfiles de ácidos grasos de los aceites se encontró un elevado número de instauraciones en su composición, lo cual tendría repercusiones negativas en las propiedades fisicoquímicas y en el desempeño en la combustión del biodiésel. El aceite de la microalga Chlorella sp. presentó una distribución de ácidos grasos más uniforme y con menos insaturaciones, en comparación con el aceite de la microalga $D$. salina, lo que podría significar mejores propiedades de lubricación, altos puntos de ebullición y poderes caloríficos, entre otras, bajo ciertas condiciones de almacenamiento y conservación del aceite.

\section{REFERENCIAS}

[1] R. Halim, M. K. Danquah and P. A. Webley, "Extraction of oil from microalgae for biodiesel production: A review", Biotech. Adv., vol. 30, pp. 709-732, May.-Jun. 2012.

[2] E. Sánchez, K. Ojeda, M. El-Halwagi et al., "Biodiesel from microalgae oil production in two sequential esterification/transesterification reactors: Pinch analysis of heat integration", Chem. Eng. J., vol. 176-177, pp. 211-216, Dec. 2011.

[3] H. Li, Z. Liu, Y. Zhang et al., "Conversion efficiency and oil quality of low-lipid highprotein and high-lipid low-protein microalgae via hydrothermal liquefaction", Bioresource Technology, vol. 154, pp. 322-329, Feb. 2014. 
[4] Y. Peralta-Ruiz, A.D. González-Delgado and V. Kafarov, "Evaluation of alternatives for microalgae oil extraction based on exergy analysis", Applied Energy, vol. 101, pp. 226236, Jan. 2013.

[5] T. Mata,A. Martins and N. Caetano, "Microalgae for biodiesel production and other applications: A review", Renewable and Sustainable Energy Reviews, vol. 14, pp. 217-232, Jan. 2010.

[6] G. Tüccar and K. Aydın, "Evaluation of methyl ester of microalgae oil as fuel in a diesel engine", Fuel, vol. 112, pp. 203-207, Oct. 2013.

[7] C. Yoo, S.Y. Jun, J. Y. Lee et al., "Selection of microalgae for lipid production under high levels carbon dioxide", Bioresource Technology, vol. 101, pp. S71-S74, Jan. 2010.

[8] J. Y. Lee, C. Yoo, S. Y. Jun et al., "Comparison of several methods for effective lipid extraction from microalgae", Bioresource Technology, vol. 101, pp. S75-S77, Jan. 2010.

[9] Y. M. Dai, K. T. Chen and C. C. Chen, "Study of the microwave lipid extraction from microalgae for biodiesel production", Chem. Eng. J., vol. 250, pp. 267-273, Aug. 2014.

[10] C. M. Teixeira and E. Morales, "Microalga como matéria-prima para a produção de biodiesel," Annals of I Congress of the Brazilian Network of Biodiesel Technology, Brasilia, 2006.

[11] C. Safi, B. Zebib, O. Merah et al., "Morphology, composition, production, processing and applications of Chlorella vulgaris: A review", Renewable and Sustainable Energy Reviews, vol. 35, pp. 265-278, Jul. 2014.

[12] P. Lamers, M. Janssen, R. De Vos et al., "Carotenoid and fatty acid metabolism in nitrogen-starved Dunaliella salina, a unicellular green microalga", $J$. of Biotech., vol. 162, pp. 21-27, Nov. 2012.

[13] P.R. Walne, "The Culture of Bivalve Molluscs: 50 Years of Experience at Conwy", Fishing News, West Byfleet, pp. 173, 1974.

[14] S. Archanaa, S. Moise and G.K. Suraishkumar, "Chlorophyll interference in microalgal lipid quantification through the Bligh and Dyer method", Biomass and Bioenergy, vol. 46, pp. 805-808, Nov. 2012.

[15] NORMA NTC 218. Grasas y aceites vegetales y animales. Determinación del índice de acidez y de la acidez.
[16] NORMA NTC 236. Grasas y aceites vegetales y animales. Determinación del índice de peróxido.

[17] NORMA NTC 287. Grasas y aceites animales y vegetales. Determinación del contenido de humedad y materia volátil.

[18] NORMA NTC 289. Grasas y aceites animales y vegetales. Determinación del índice de refracción.

[19] NORMA NTC 336. Grasas y aceites animales y vegetales. Método de la determinación de la densidad.

[20] NORMA NTC 5478. Grasas y aceites comestibles. Determinación del punto humo, chispa e ignición método Cleveland de copa abierta.

[21] N. Aguirre, J. Palacio, I. Correa et al., "Ensayos de bioestimulación algal con diferentes relaciones nitrógeno: fósforo, bajo condiciones de laboratorio", Revista de Ingenierías, Universidad de Medellín, vol. 6, pp. 11-21, Jul.Dic. 2007.

[22] R. Salomón, I. Albarracín and G. Pio, "Sensibilidad de Chlorella vulgaris y Scenedesmus quadricauda a la Cipermetrina. Fase preliminar", Retel, vol. 7, pp. 1-15, May. 2005.

[23] V. Díaz and C. Ordóñez, C. "Evaluación del pH y la agitación del medio más adecuado para el crecimiento de la Dunaliella salina en condiciones de laboratorio", Tesis de Grado Microbiología Industrial, Pontificia Universidad Javeriana, Bogotá, 2006.

[24] D. Fimbres, L. Mercado, A. Murguía et al., "Crecimiento y biomasa de Dunaliella sp. cultivada en medios limitantes en nitrógeno", Biotecnia, vol. 12, pp. 58-66, Sept.-Dic. 2010.

[25] A. Vásquez-Suárez, M. Guevara, G. Salazar et al., "Crecimiento y composición bioquímica de cuatro especies de Dunaliella para ser utilizadas en Acuicultura", Boletín del Centro de Investigaciones Biológicas de la Universidad de Zulia, vol. 41, pp. 181-194, 2007.

[26] L. Rodolfi, G. Chini-Zittelli, N. Bassi et al., "Microalgae for oil: Strain selection, induction of lipid synthesis and outdoor mass cultivation in a low-cost photobioreactor", Biotechnology and Bioengineering, vol. 102, pp. 100-112, Jan. 2009.

[27] Y. Liang, N. Sarkany and Y. Cui, "Biomass and lipid productivities of Chlorella vulgaris under 
autotrophic, heterotrophic and mixotrophic growth conditions", Biotechnology Letters, vol. 31, pp. 1043-1049, Jul. 2009.

[28] R. Praveenkumar, B. Kim, E. Choi, K. Lee, J. Park, J. Lee, Y. Lee, Y. Oh, "Improved biomass and lipid production in a mixotrophic culture of Chlorella sp. KR-1 with addition of coal-fired flue-gas", Bioresource Technology, vol. 171, pp. 500-505, Nov. 2014.

[29] R. Praveenkumar, K. Shameera, G. Mahalakshmi, M. Akbarsha, N. Thajuddin, "Influence of nutrient deprivations on lipid accumulation in a dominant indigenous microalga Chlorella sp., BUM11008: Evaluation for biodiesel production", Biomass and Bioenergy, vol. 37, pp. 60-66, Feb. 2012.

[30] P. Zhao, X. Yu, J. Li, X. Tang, Z. Huang, "Enhancing lipid productivity by co-cultivation of Chlorella sp. U4341 and Monoraphidium sp. FXY-10", Journal of Bioscience and Bioengineering, vol. 118, pp. 72-77, Jul. 2014.

[31] L. Gouveia and A.C. Oliveira, "Microalgae as a raw material for biofuels production", Journal of Industrial Microbiology \& Biotechnology, vol. 36, pp. 269-274, Feb. 2009.

[32] M. Takagi and K., T. Yoshida, "Effect of salt concentration on intracellular accumulation of lipids and triacylglyceride in marine microalgae Dunaliella cells", Journal of Bioscience and Bioengineering, vol. 101, pp. 223-226, Mar. 2006.

[33] G. Petkov and G. Garcia, "Which are fatty acids of the green alga Chlorella?", Biochemical Systematics and Ecology, vol. 35, pp. 281-285, May. 2007.

[34] D. Leung, W. Xuan and M. Leung, "A review on biodiesel production using catalyzed transesterification", Applied Energy, vol. 87, pp. 1083-1095, Apr. 2010. 